\title{
Recovery of a high-pressure phase formed under laser-driven compression
}

\author{
M. G. Gorman $\odot,{ }^{1, *}$ D. McGonegle, ${ }^{2}$ S. J. Tracy, ${ }^{3}$ S. M. Clarke, ${ }^{1}$ C. A. Bolme $\odot,{ }^{4}$ A. E. Gleason $\odot,{ }^{5}$ \\ S. J. Ali $\odot,{ }^{1}$ S. Hok, ${ }^{5}$ C. W. Greeff $\odot,{ }^{6}$ P. G. Heighway $\odot,{ }^{2}$ K. Hulpach, ${ }^{7}$ B. Glam, ${ }^{8}$ E. Galtier, ${ }^{9}$ \\ H. J. Lee, ${ }^{9}$ J. S. Wark $\odot,{ }^{2}$ J. H. Eggert, ${ }^{1}$ J. K. Wicks, ${ }^{7}$ and R. F. Smith $\oplus^{1}$ \\ ${ }^{1}$ Lawrence Livermore National Laboratory, 7000 East Avenue, Livermore, California 94500, USA \\ ${ }^{2}$ Department of Physics, Clarendon Laboratory, Parks Road, University of Oxford, Oxford OX1 3PU, United Kingdom \\ ${ }^{3}$ Earth and Planets Laboratory, Carnegie Institution of Washington, Washington DC 20015, USA \\ ${ }^{4}$ Shock and Detonation Physics, Los Alamos National Laboratory, P.O. Box 1663, Los Alamos, New Mexico 87545, USA \\ ${ }^{5}$ Stanford Institute for Materials and Energy Sciences, SLAC National Accelerator Laboratory, Menlo Park, California 94025, USA \\ ${ }^{6}$ Los Alamos National Laboratory, P.O. Box 1663, Los Alamos, New Mexico 87545, USA \\ ${ }^{7}$ Department of Earth and Planetary Sciences, Johns Hopkins University, Baltimore, Maryland 21218, USA \\ ${ }^{8}$ Soreq Nuclear Research Center, Yavne 81800, Israel \\ ${ }^{9}$ Linac Coherent Light Source, SLAC National Accelerator Laboratory, Menlo Park, California 94025, USA
}

(Received 14 April 2020; revised 9 June 2020; accepted 12 June 2020; published 6 July 2020)

\begin{abstract}
The recovery of metastable structures formed at high pressure has been a long-standing goal in the field of condensed matter physics. While laser-driven compression has been used as a method to generate novel structures at high pressure, to date no high-pressure phases have been quenched to ambient conditions. Here we demonstrate, using in situ x-ray diffraction and recovery methods, the successful quench of a high-pressure phase which was formed under laser-driven shock compression. We show that tailoring the pressure release path from a shock-compressed state to eliminate sample spall, and therefore excess heating, increases the recovery yield of the high-pressure $\omega$ phase of zirconium from $0 \%$ to $48 \%$. Our results have important implications for the quenchability of novel phases of matter demonstrated to occur at extreme pressures using nanosecond laser-driven compression.
\end{abstract}

DOI: 10.1103/PhysRevB.102.024101

\section{INTRODUCTION}

High-pressure synthesis is a versatile tool for the creation of novel phases of matter with unique chemical and physical properties [1]. Such high-pressure materials have potential for revolutionary technological advances if they can be recovered to ambient conditions. The ability to synthesize bulk quantities of the diamond structure of carbon is perhaps the best example of how a metastable phase can be recovered to ambient pressure and have tremendous utility in industry [2]. Indeed, the recovery of metastable phases formed at high pressure has been demonstrated with great success in static compression experiments [3-6] as well as dynamic compression experiments which used gas gun drivers $[7,8]$ and, more recently, laser-induced microexplosions [9]. Theoretical predictions now show that many novel forms of materials exist at ultrahigh pressures [10-12], with some predicted to be quenchable to ambient conditions, such as the $\mathrm{BC} 8$ phase of carbon, which is predicted to form at 10 million atm and be stronger than diamond [13]. Such extreme conditions of pressure are beyond the limits that can be achieved through static compression methods or dynamic compression using gas guns but can be readily achieved through laser-driven compression (LDC) [14].

*gorman11@1lnl.gov
Laser drivers therefore represent the best prospect for recovering exotic forms of matter now predicted to exist at ultrahigh pressures. In addition, LDC is advantageous for synthesizing novel phases, as the rapid compression rates achievable can facilitate access to nonequilibrium states at high pressures and temperatures [15,16]. In many cases, phase transformations observed in LDC experiments show large hysteresis, particularly when the time scale of pressure release is comparable to the kinetic time scales associated with the transition [17]. Indeed, several recent LDC studies have observed the existence of high-pressure phases at zero pressure for tens of nanoseconds on shock release [16,1820], but to date there have been no reported cases where recovery was achieved. Here we report x-ray diffraction measurements of elemental zirconium ( $\mathrm{Zr}$ ) on shock release from $22 \mathrm{GPa}$ which demonstrate the successful quenching of a high-pressure phase after LDC. We identified that tailoring the sample release path to minimize spall, and therefore excess heating, was key to successfully recovering a high-pressure omega $(\omega)$ phase of Zr. Our results herald the possibility of recovering novel phases of matter formed at ultrahigh pressure using LDC.

Zirconium has been studied extensively both theoretically [21-23] and experimentally [24-26] as a model system for understanding phase transformation kinetics. Under ambient conditions, $\mathrm{Zr}$ exists in the hexagonally close-packed alpha $(\alpha)$ phase (space-group symmetry $\mathrm{P} 6_{3} / m m c, Z=2$ ). 
Under quasistatic compression at room temperature, a transformation to the hexagonal $\omega$ phase (space-group symmetry P $6 / \mathrm{mmm}, Z=3$ ) occurs at $3 \mathrm{GPa}$, before a further transformation to a body-centered cubic, beta $(\beta)$ phase (space-group symmetry I $m-3 m, Z=2$ ) at $30 \mathrm{GPa}$ [26]. The $\alpha \rightarrow \omega$ transformation has been shown to exhibit strong hysteresis, as the $\omega$ phase has been recovered to ambient conditions in previous static and gas gun experiments $[7,24,27]$. The strong phase transformation kinetics are due to the reconstructive nature of the $\omega \rightarrow \alpha$ transformation, which occurs through a shuffle and shear mechanism [28,29]. The $\omega$ phase of $\mathrm{Zr}$ is therefore the ideal candidate to demonstrate the feasibility of recovering a metastable phase using LDC.

\section{EXPERIMENTAL DETAILS}

To study the $\omega \rightarrow \alpha$ transformation on shock release, we performed LDC experiments at the Matter in Extreme Conditions (MEC) end station at the Linac Coherent Light Source (LCLS). In each experiment, a 500- $\mu$ m-diameter laser spot ablatively drove a shock wave into the $\mathrm{Zr}$ sample using a 10-ns flat-top laser pulse. The sample was subsequently probed after a predetermined time delay using the 50-fs LCLS x-ray beam. When the laser drive turns off, a forward-propagating pressure release wave transits the target assembly [30]. A schematic of the experimental setup and target design is shown in Fig. 1(a). The target consisted of a $50-\mu \mathrm{m}$ polyimide-corrundum ablator bonded to a $25-\mu \mathrm{m}$ polycrystalline $\mathrm{Zr}$ foil. The velocity history of each target was measured by the line-imaging Velocity Interferometry System for Any Reflector (VISAR) [31] at MEC. Typical raw VISAR data and the corresponding velocity line-out obtained at MEC are shown in Fig. 1(b). The high-brilliance LCLS x-ray beam delivered up to $\sim 10^{12} 11.2-\mathrm{keV}$ photons over $50 \mathrm{fs}$ with $\Delta E / E=0.5 \%$, and diffraction was collected by CornellStanford Pixel Array Detectors (CSPADs), positioned behind the sample. Raw two-dimensional (2D) diffraction data from each CSPAD were integrated azimuthally to produce a $1 \mathrm{D}$ intensity line-out as shown in Fig. 1(c).

\section{IN SITU X-RAY DIFFRACTION EXPERIMENTS AT MEC}

A series of $\mathrm{Zr}$ samples was shock-compressed to a peak pressure of $22 \mathrm{GPa}$, using identical laser conditions in each experiment, which were sufficient to ensure complete transformation to the high-pressure $\omega$ phase. After shock transit through the $\mathrm{Zr}$ sample and arrival at the free surface, strong pressure release waves propagate back into the sample [30]. The time delay of the x-ray probe relative to shock breakout was increased with each subsequent experiment so as to capture the phase behavior of $\mathrm{Zr}$ as the pressure conditions rapidly reverted from the $\omega$ to the $\alpha$ stability fields. A diffraction snapshot taken $1 \mathrm{~ns}$ after shock breakout showed diffraction from the high-pressure $\omega$ phase with volumetric compression $\frac{V}{V_{0}}=0.838$, which is consistent with a shock pressure of $22 \mathrm{GPa}$ [22]. The refined lattice parameters were $a=4.783 \AA$ and $c=2.958 \AA$. Diffraction from the $\alpha$ phase was very weak, indicating that much of the sample remained in the peak pressure state soon after breakout [Fig. 2(a), i]. Additional diffraction peaks were observed due to the presence of $\mathrm{Al}_{2} \mathrm{O}_{3}$ (a)

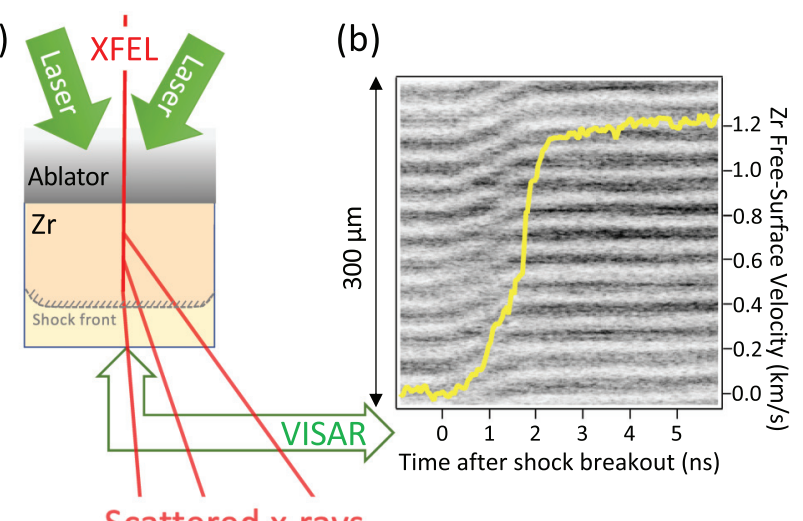

(c)
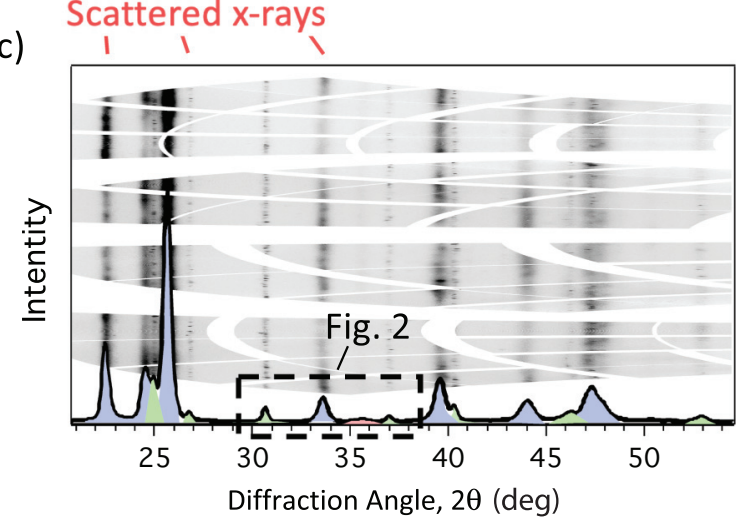

FIG. 1. (a) Experimental setup at MEC. An intense laser pulse drove an ablatively driven shock wave into the target package, which consisted of a polyimide ablator bonded to a polycrystalline $\mathrm{Zr}$ foil. The sample was probed by the collimated LCLS x-ray beam and scattered diffraction data were collected in a transmission geometry. The $\mathrm{Zr}$ free surface velocity history was recorded by the velocity interferometry (VISAR) diagnostic. (b) Typical raw VISAR data with the analyzed velocity profile overlaid. (c) Typical 2D diffraction data, with diffraction maxima warped into lines of constant $2 \theta$, with the corresponding, azimuthally integrated $1 \mathrm{D}$ diffraction profile overlaid. Shading under the peaks refers to the identified phase (see Fig. 2).

dispersed in the polyimide ablator. The diffraction patterns show a clear shifting of $\omega$ diffraction peaks to lower $2 \theta$ with increasing delay time (Fig 2(a) ii-iii) demonstrating that the $\omega$ lattice had expanded on decompression. In addition, the intensity of diffraction peaks of the $\alpha$ phase increased relative to the $\omega$ peaks as the sample underwent the reverse transformation. After $30 \mathrm{~ns}$, pressure had been completely released in the sample yet diffraction from both the $\alpha$ and the $\omega$ phases was still observed. Diffraction from the $\omega$ phase at this time indicated that the $\omega$ lattice had expanded $\sim 17 \%$ $\left(\frac{V}{V_{0}}=1.005\right)$ relative to the peak pressure state.

The sample volumes of $\omega-\mathrm{Zr}$ and $\alpha-\operatorname{Zr}\left(\frac{V}{V_{0}} \alpha=1.021\right)$ and corrundum $\left(\frac{V}{V_{0}} \mathrm{Al}_{2} \mathrm{O}_{3}=1.015\right)$ at this time all indicated thermal expansion due to the samples' existing at an elevated temperature and zero pressure. The determined sample volumes remained approximately constant with increasing XFEL delay [Fig. 2(a), iv-vi], indicating that the $\omega$ phase persisted at ambient pressure and elevated temperature until at least $150 \mathrm{~ns}$ after breakout before complete reversion to the $\alpha$ phase was observed at $250 \mathrm{~ns}$ [Fig. 2(a), vii]. 
(a)

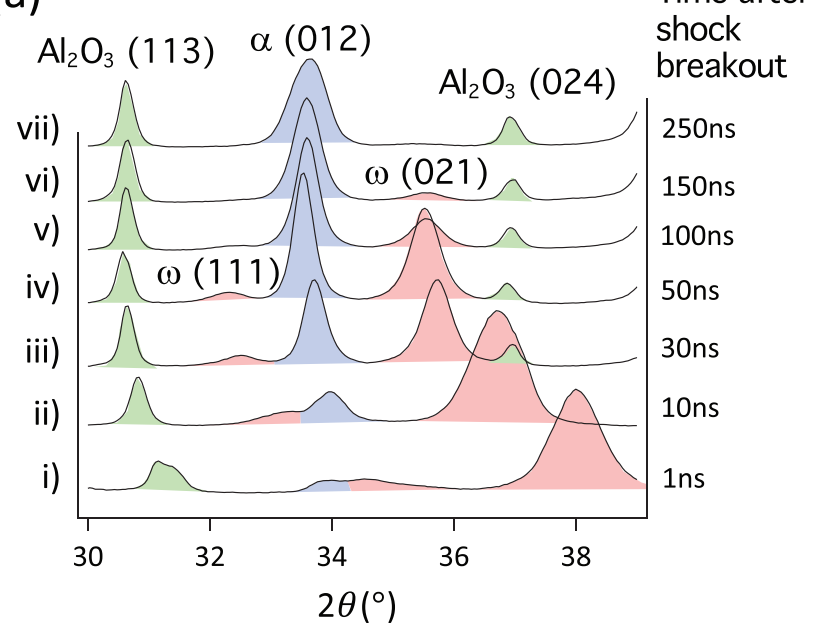

(b)

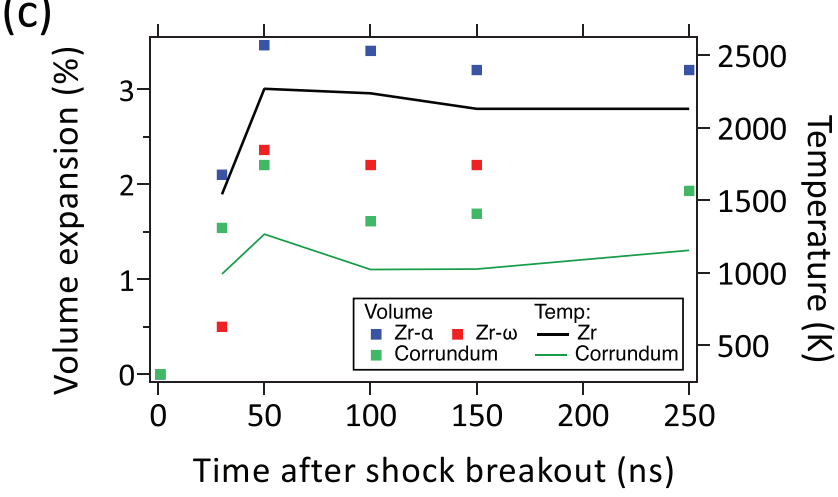

FIG. 2. (a) Waterfall plot of diffraction data collected at MEC after shock breakout and subsequent rapid pressure release from $22 \mathrm{GPa}$. The time in each case indicates the XFEL delay time relative to shock breakout at the $\mathrm{Zr}$ free surface. Specific diffraction peaks from the $\alpha$ phase (blue), $\omega$ phase (red), and corrundum phase from the ablator (green) are labeled. (b) Relative volume fraction of the $\alpha$ (blue symbols) and $\omega$ (red symbols) phases as a function of the time after shock breakout. When releasing from a free surface, the $\mathrm{Zr}$ sample, initially in the $\omega$ phase, undergoes full reversion to the $\alpha$ phase within $250 \mathrm{~ns}$. The transformation rate is fit well with an exponential decay/growth (solid lines). (c) Volume expansion of the sample as a function of the time after shock breakout (symbols) and corresponding deduced temperature (solid lines). The $\mathrm{Zr}$ sample temperature is determined to exceed $2000 \mathrm{~K}$ on release, significantly hotter than the corundum ablator, due to the occurrence of spall and fracture of the $\mathrm{Zr}$ sample.
We performed Rietveld analysis of the diffraction data, which allowed for quantitative determination of the $\alpha$ and $\omega$ volume fractions as a function of the shock breakout [Fig. 2(b)]. The data show that the volume fraction of the high-pressure $\omega$ followed an exponential decay, with the final $10 \%$ converting back to $\alpha-\mathrm{Zr}$ in $\sim 100 \mathrm{~ns}$. As well as the volume fraction in situ, we also deduced quantitative sample temperatures as a function of the shock breakout from the measured thermal expansion of the sample [Fig. 2(c)]. Indeed, the Mie-Gruneisen-Debye equation of states constructed for corundum [32] and $\alpha-\mathrm{Zr}$ [27] showed that the corundum/ablator (volume expansion, $\sim 2 \%$ ) existed at temperatures of $\sim 1100 \mathrm{~K}$ on average for $250 \mathrm{~ns}$ after breakout, while the $\mathrm{Zr}$ sample (volume expansion of $\alpha-\mathrm{Zr}, \sim 3 \%$ ) existed at temperatures exceeding $2000 \mathrm{~K}$ on average. The metastable $\omega$ phase is well known to become highly unfavorable relative to the stable $\alpha$ phase at elevated temperatures, which explains why complete reversion was observed in our MEC experiments [28,33-35]. While it has recently been reported that the early stages of rapid release from high pressure at a free surface can be highly nonisentropic due to plastic work heating induced by material strength [36], the level of sample heating in our experiments [Fig. 2(c)] was unexpected [37] and indicated an additional source of heating, which inhibited the recovery of the high-pressure $\omega$ phase.

To investigate, we modeled the hydrodynamic flow of pressure waves in the sample by matching 1D Lagrangian simulations to the MEC experimental conditions. Our simulations showed that the release of sample pressure after shock compression occurs via the interaction of forward- and backwardpropagating release waves (see Supplemental Movie 1 [30]) which coalesce within the $\mathrm{Zr}$ sample. Such interactions led to negative stresses in the sample which exceeded the spall strength [37]. Spallation has been shown in various metals to generate significant, irreversible sample heating due to viscoplastic deformation of the sample [38-40]. We therefore hypothesized that spallation had occurred during our MEC experiments and caused heating of the $\mathrm{Zr}$ samples to temperatures sufficient to ensure full reversion to the $\alpha$ phase.

In order to eliminate this unwanted source of heating, the interaction of sample release waves would have to be altered to eliminate the occurrence of spallation. Our hydrocode modeling showed that a tamper of similar impedance to $\mathrm{Zr}$, such as LiF, would greatly reduce the amplitude of the backwardpropagating release wave and, therefore, virtually eliminate negative stresses experienced by the sample (see Supplemental Movie 2 [30]). These findings suggested that Zr samples with a high-impedance tamper would undergo a more isentropic release path and exist at temperatures which may be low enough to prevent full phase reversion. Indeed the release path temperature, assuming purely isentropic release from $22 \mathrm{GPa}$, is estimated to be $\sim 450 \mathrm{~K}[22]$, significantly lower than the sample temperatures deduced from our MEC experiments.

\section{RECOVERY EXPERIMENTS AT THE JUPITER LASER FACILITY}

To confirm these hypotheses, we performed additional experiments at the Jupiter Laser Facility which tested the free surface and LiF target designs under drive conditions 
(a)

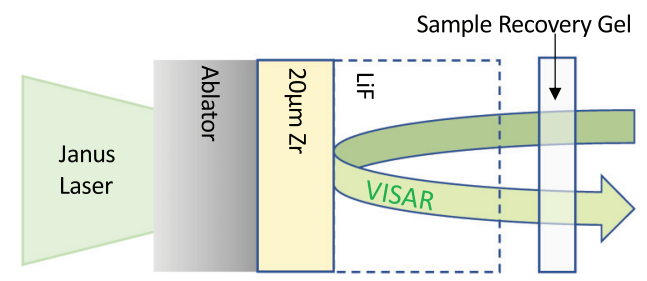

(b)

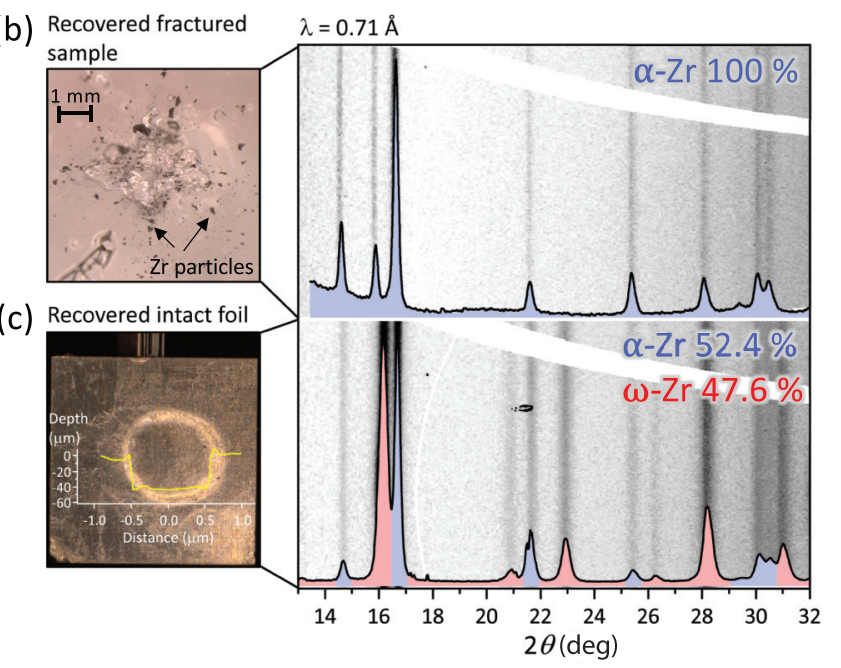

FIG. 3. (a) Target design for recovery experiments at the Jupiter Laser Facility. The dashed line indicates that in some cases a high-impedance LiF tamper was used. (b) Photograph of recovered $\mathrm{Zr}$ material which had been collected in the sample recovery gel. Multiple spall events resulted in an average $\mathrm{Zr}$ particle size of $\sim 10 \mu \mathrm{m}$. Postshot diffraction measurements of this material showed only diffraction from the $\alpha-\mathrm{Zr}$ phase, consistent with the MEC experiments. Raw 2D diffraction data are shown with the azimuthally integrated profile overlaid. (c) Postshot photograph of the recovered $\mathrm{Zr}$ material which released into a LiF window. Profilometry measurements of the intact foil show that the shocked region was $1 \mathrm{~mm}$ in diameter and had a depth of $40 \mu \mathrm{m}$. Raw 2D diffraction data are shown with the azimuthally integrated profile overlaid. A two-phase Reitveld refinement of these diffraction data showed that the volume fractions of the $\omega-\mathrm{Zr}$ (red shading) and $\alpha-\mathrm{Zr}$ (blue shading) phases were $47.6 \%$ and $52.4 \%$, respectively.

similar to those at MEC. The aim of these experiments was to collect the released target material in each case and perform x-ray diffraction measurements ex situ. A detailed discussion of the experimental setup can be found in [41]. A transparent recovery gel was placed along the VISAR optical axis in close proximity to the rear of the target to collect forward-propagating debris [Fig. 3(a)]. Recovered material demonstrated that the $\mathrm{Zr}$ sample, when releasing from a free surface, broke up through multiple fracture events into particles of order $10 \mu \mathrm{m}$ [Fig. 3(b)]. These findings indicated that spallation and fracture had occurred in our MEC experiments as predicted by our hydrocode simulations. The x-ray diffraction measurements on these samples confirmed that full reversion to the $\alpha$ phase occurred, consistent with our in situ measurements from MEC [Fig. 3(b)].

In contrast, when releasing into a $\mathrm{LiF}$ tamper we observed that the $\mathrm{Zr}$ sample remained intact [Fig. 3(c)], confirming predictions that spallation can be eliminated by tailoring the pressure release path of the sample. The shocked region of the $\mathrm{Zr}$ foil is identified by a $1-\mathrm{mm}$ circular depression of the surface of $\sim 40 \mu \mathrm{m}$ [Fig. 3(c)]. Remarkably, Rietveld analysis of x-ray diffraction measurements of the recovered sample indicated that $48 \%$ of the high-pressure $\omega$ phase had been successfully recovered. The absence of spall within the $\mathrm{LiF}$ sample and the associated heating due to viscoplastic deformation are shown to dramatically reduce the $\omega \rightarrow \alpha$ reversion rate.

\section{DISCUSSION}

The kinetics of phase transformations have often been modeled using simple phenomenological rate equations such as the Johnson-Mehl-Avrami-Kolmogorov (JMAK) model [42-44]. Such models have been used successfully to describe the recrystallization of materials from melt $[45,46]$ but have also been applied to solid-solid phase transformations [47]. The volume fraction of material which has transformed after a certain time is governed by a rate constant $k$, which is assumed to follow simple Arrhenius behavior of the form

$$
k=\mathrm{A} \exp \left(-\frac{E_{A}}{k_{B} T}\right),
$$

where $\mathrm{A}$ is a constant, $E_{A}$ is the activation energy (in $\left.\mathrm{eV}\right), k_{B}$ is the Boltzmann constant (in $\mathrm{eV} / \mathrm{K}$ ) and $T$ is the temperature (in K). Applying this simple model to our data reveals that the rate of transformation $k$ in the free surface experiments, where $T \sim 2000 \mathrm{~K}$, compared with the LiF experiments, where $T \sim$ $500 \mathrm{~K}$, is calculated to be 12 orders of magnitude higher $\left(E_{A}\right.$ of the $\omega \rightarrow \alpha$ transition taken to be $\sim 1.5 \mathrm{eV}$ [28]).

Recent LDC experiments have observed the temporary existence of metastable polymorphs under ambient conditions in $\mathrm{Bi}, \mathrm{Sb}$, and $\mathrm{SiC}[16,19,20]$. Crucially, all samples in these experiments underwent free surface release, suggesting that the metastable phases observed may be readily recovered with more judicious tailoring of the sample release path as demonstrated in our experiments. The impedance of the tamper layer can be altered to better match that of the sample to minimize the amplitude of the rear surface release waves entering the sample, which would eliminate the occurrence of spall. $A b$ initio simulations can now predict novel forms of matter which crystallize far away from equilibrium under extreme conditions of pressure and temperature [9-11] and guide experiments towards the candidates which could be recovered to ambient conditions [13]. The BC8 phase of carbon is predicted to form at $1000 \mathrm{GPa}$ and be metastable on pressure release to ambient conditions [13]. Such pressures are now routinely achievable at the highest-power laser facilities. By designing both the laser pulse and the target to control the sample pressure release so as to avoid excessive heating (and perhaps melting), such a metastable phase may be able to be recovered for the first time.

While only around $1 \mu \mathrm{g}$ of metastable material is recovered in each of the current experiments, next-generation laser facilities have the capability to perform experiments at $10 \mathrm{~Hz}$ [48], which will vastly increase the volume of material that can be recovered. The recovered material could also potentially be 
used as a seed material to synthesize larger quantities using established techniques [49].

\section{CONCLUSION}

We report the successful recovery of a metastable phase of matter which was formed under LDC. In situ X-ray diffraction techniques coupled with diffraction measurements of recovered samples demonstrated that the high-pressure $\omega$ phase of $\mathrm{Zr}$ underwent complete reversion to the stable $\alpha$ phase on release from a free surface but was recovered $(48 \% \omega: 52 \%$ $\alpha$ ) when a high-impedance $\mathrm{LiF}$ tamper was used. Wave reverberations in the free surface experiments caused the sample to undergo spallation and fracture, which caused significant sample heating as deduced by the measured thermal expansions and ultimately aided the reverse transformation to the $\alpha$ phase. These results demonstrate the feasibility of high-pressure recovery with laser compression and show how this method can complement existing high-pressure methods in their search for revolutionary functional materials. Crucially, our results pave the way for unprecedented efforts to recover novel materials at pressures above 10 million atm.

\section{ACKNOWLEDGMENTS}

Lawrence Livermore National Laboratory is operated by Lawrence Livermore National Security (LLNS), LLC, for the U.S. Department of Energy, National Nuclear Security Administration, under Contract No. DE-AC52 07NA27344. J.S.W. would like to acknowledge support from the Engineering and Physical Sciences Research Council under Grants No. EP/J017051/1 and No. EP/S025065/1. D.M. was supported by LLNS under Contract No. B595954. C.A.B. would like to acknowledge support from Science Campaign 2 at Los Alamos National Laboratory, which is operated for the National Nuclear Security Administration of the U.S. Department of Energy under Contract No. DE-AC52-06NA25396. Use of the Linac Coherent Light Source (LCLS), SLAC National Accelerator Laboratory, was supported by the U.S. Department of Energy, Office of Science, Office of Basic Energy Sciences, under Contract No. DE-AC02-76SF00515. The MEC instrument is supported by the U.S. Department of Energy, Office of Science, Office of Fusion Energy Sciences, under Contract No. SF00515. We thank Gaia Righi for introducing the transparent sample recovery gel concept for our Jupiter Laser Facility experiments. We gratefully acknowledge Connor Krill for providing the framework to produce movies of our hydrodynamic simulation outputs.
[1] L. Zhang, Y. Wang, J. Lv, and Y. Ma, Nat. Rev. Mater. 2, 17013 (2017).

[2] T. Evans and P. James, Proc. R. Soc. London A 277, 260 (1964).

[3] R. Wentorf and J. Kasper, Science 139, 338 (1963).

[4] U. Schwarz, S. Tence, O. Janson, C. Koz, C. Krellner, U. Burkhardt, H. Rosner, F. Steglich, and Y. Grin, Angew. Chem. Int. Ed. 52, 9853 (2013).

[5] S. M. Clarke, M. Amsler, J. P. Walsh, T. Yu, Y. Wang, Y. Meng, S. D. Jacobsen, C. Wolverton, and D. E. Freedman, Chem. Mater. 29, 5276 (2017).

[6] S. M. Clarke, J. P. Walsh, M. Amsler, C. D. Malliakas, T. Yu, S. Goedecker, Y. Wang, C. Wolverton, and D. E. Freedman, Angew. Chem. Int. Ed. 55, 13446 (2016).

[7] E. K. Cerreta, F. L. Addessio, C. A. Bronkhorst, D. W. Brown, J. P. Escobedo, S. J. Fensin, G. T. G. Iii, T. Lookman, P. A. Rigg, and C. P. Trujillo, J. Phys. Conf. Ser. 500, 032003 (2014).

[8] D. R. Jones, B. M. Morrow, C. P. Trujillo, G. T. Gray III, and E. K. Cerreta, J. Appl. Phys. 122, 045902 (2017).

[9] L. Rapp, B. Haberl, C. J. Pickard, J. E. Bradby, E. G. Gamaly, J. S. Williams, and A. V. Rode, Nat. Commun. 6, 7555 (2015).

[10] M. Martinez-Canales, C. J. Pickard, and R. J. Needs, Phys. Rev. Lett. 108, 045704 (2012).

[11] C. J. Pickard and R. J. Needs, Nat. Mater. 9, 624 (2010).

[12] P. Li, G. Gao, Y. Wang, and Y. Ma, J. Phys. Chem. C 114, 21745 (2010).

[13] J. Sun, D. D. Klug, and R. Martoňák, J. Chem. Phys. 130, 194512 (2009).

[14] R. F. Smith, J. H. Eggert, R. Jeanloz, T. S. Duffy, D. G. Braun, J. R. Patterson, R. E. Rudd, J. Biener, A. E. Lazicki, A. V. Hamza, J. Wang, T. Braun, L. X. Benedict, P. M. Celliers, and G. W. Collins, Nature 511, 330 (2014).
[15] M. G. Gorman, A. L. Coleman, R. Briggs, R. S. McWilliams, D. McGonegle, C. A. Bolme, A. E. Gleason, E. Galtier, H. J. Lee, E. Granados, M. Śliwa, C. Sanloup, S. Rothman, D. E. Fratanduono, R. F. Smith, G. W. Collins, J. H. Eggert, J. S. Wark, and M. I. McMahon, Sci. Rep. 8, 16927 (2018).

[16] A. L. Coleman, M. G. Gorman, R. Briggs, R. S. McWilliams, D. McGonegle, C. A. Bolme, A. E. Gleason, D. E. Fratanduono, R. F. Smith, E. Galtier, H. J. Lee, B. Nagler, E. Granados, G. W. Collins, J. H. Eggert, J. S. Wark, and M. I. McMahon, Phys. Rev. Lett. 122, 255704 (2019).

[17] R. F. Smith, J. H. Eggert, D. C. Swift, J. Wang, T. S. Duffy, D. G. Braun, R. E. Rudd, D. B. Reisman, J. P. Davis, M. D. Knudson, and G. W. Collins, J. Appl. Phys. 114, 223507 (2013).

[18] D. Kraus, J. Vorberger, A. Pak, N. J. Hartley, L. B. Fletcher, S. Frydrych, E. Galtier, E. J. Gamboa, D. O. Gericke, S. H. Glenzer, E. Granados, M. J. MacDonald, A. J. MacKinnon, E. E. McBride, I. Nam, P. Neumayer, M. Roth, A. M. Saunders, A. K. Schuster, P. Sun, T. van Driel, T. Döppner, and R. W. Falcone, Nature Astron. 1, 606 (2017).

[19] S. J. Tracy, S. J. Turneaure, and T. S. Duffy, Phys. Rev. Lett. 120, 135702 (2018).

[20] M. G. Gorman, A. L. Coleman, R. Briggs, R. S. McWilliams, A. Hermann, D. McGonegle, C. A. Bolme, A. E. Gleason, E. Galtier, H. J. Lee, E. Granados, E. E. McBride, S. Rothman, D. E. Fratanduono, R. F. Smith, G. W. Collins, J. H. Eggert, J. S. Wark, and M. I. McMahon, Appl. Phys. Lett. 114, 120601 (2019).

[21] I. Schnell and R. C. Albers, J. Phys.: Condens. Matter 18, 1483 (2006).

[22] C. W. Greeff, Model. Simul. Mater. Sci. Eng. 13, 1015 (2005).

[23] H. Zong, G. Pilania, X. Ding, G. J. Ackland, and T. Lookman, npj Comput. Mater. 4, 48 (2018). 
[24] J. C. Jamieson, Science 140, 72 (1963).

[25] Y. K. Vohra, J. Nucl. Mater. 75, 288 (1978).

[26] J. Zhang, Y. Zhao, C. Pantea, J. Qian, L. L. Daemen, P. A. Rigg, R. S. Hixson, C. W. Greeff, G. T. Gray III, Y. Yang, L. Wang, Y. Wang, and T. Uchida, J. Phys. Chem. Solids 66, 1213 (2005).

[27] Y. Zhao, J. Zhang, C. Pantea, J. Qian, L. L. Daemen, P. A. Rigg, R. S. Hixson, G. T. Gray III, Y. Yang, L. Wang, Y. Wang, and T. Uchida, Phys. Rev. B 71, 184119 (2005).

[28] H. Zong, T. Lookman, X. Ding, C. Nisoli, D. Brown, S. R. Niezgoda, and S. Jun, Acta Mater. 77, 191 (2014).

[29] K. Bhattacharya, S. Conti, G. Zanzotto, and J. Zimmer, Nature 428, 55 (2004).

[30] See Supplemental Material at http://link.aps.org/supplemental/ 10.1103/PhysRevB.102.024101 for movies, additional information on sample metrology, and details on the analysis.

[31] P. M. Celliers, D. K. Bradley, G. W. Collins, D. G. Hicks, T. R. Boehly, and W. J. Armstrong, Rev. Sci. Instrum. 75, 4916 (2004).

[32] L. S. Dubrovinsky, S. K. Saxena, and P. Lazor, Phys. Chem. Miner. 25, 434 (1998).

[33] D. Brown, J. Almer, L. Balogh, E. Cerreta, B. Clausen, J. Escobedo-Diaz, T. Sisneros, P. Mosbrucker, E. Tulk, and S. Vogel, Acta Mater. 67, 383 (2014).

[34] T. S. E. Low, D. W. Brown, B. A. Welk, E. K. Cerreta, J. S. Okasinski, and S. R. Niezgoda, Acta Mater. 91, 101 (2015).

[35] T. S. E. Low and S. R. Niezgoda, Acta Mater. 156, 104 (2018).

[36] P. G. Heighway, M. Śliwa, D. McGonegle, C. Wehrenberg, C. A. Bolme, J. Eggert, A. Higginbotham, A. Lazicki, H. J. Lee, B. Nagler, H. S. Park, R. E. Rudd, R. F. Smith, M. J. Suggit, D.
Swift, F. Tavella, B. A. Remington, and J. S. Wark, Phys. Rev. Lett. 123, 245501 (2019).

[37] A. M. Molodets, A. A. Golyshev, D. V. Shakhrai, and D. Yu. Kovalev, Phys. Solid State 62, 65 (2020).

[38] N. Gunkelmann, E. M. Bringa, and H. M. Urbassek, J. Appl. Phys. 118, 185902 (2015).

[39] Y. Liao, M. Xiang, X. Zeng, and J. Chen, Mech. Mater. 84, 12 (2015).

[40] E. N. Hahn, S. J. Fensin, T. C. Germann, and G. T. Gray III, Acta Mater. 159, 241 (2018).

[41] R. F. Smith, C. A. Bolme, D. J. Erskine, P. M. Celliers, S. Ali, J. H. Eggert, S. L. Brygoo, B. D. Hammel, J. Wang, and G. W. Collins, J. Appl. Phys. 114, 133504 (2013).

[42] A. Kolmogorov, Bull. Acad. Sci. USSR Ser. Math. 3, 355 (1937) [in Russian].

[43] M. Avrami, J. Chem. Phys. 7, 1103 (1939).

[44] W. Johnson and R. Mehl, Trans. Metal. Soc. 135, 416 (1939).

[45] E. D. Zanotto, Thermochim. Acta 280-281, 73 (1996).

[46] M. C. Weinberg, Thermochim. Acta 280-281, 63 (1996).

[47] A. E. Gleason, C. A. Bolme, E. Galtier, H. J. Lee, E. Granados, D. H. Dolan, C. T. Seagle, T. Ao, S. Ali, A. Lazicki, D. Swift, P. Celliers, and W. L. Mao, Phys. Rev. Lett. 119, 025701 (2017).

[48] P. Phillips, S. Banerjee, J. Smith, K. Ertel, P. Mason, T. Butcher, M. D. Vido, M. Divoky, B. Costello, M. Hanus, J. Pillar, P. Navaratil, A. Lucianetti, M. Tyldesley, T. Mocek, C. Edwards, C. Hernandez-Gomez, and J. Collier, in High-Power, HighEnergy, and High-Intensity Laser Technology IV, Vol. 11033, edited by J. Hein and T. J. Butcher (International Society for Optics and Photonics, San Francisco, 2019).

[49] J. C. Angus and C. C. Hayman, Science 241, 913 (1988). 